\title{
BOUNDARIES AND POLYHEDRAL BANACH SPACES
}

\author{
V. P. FONF, R. J. SMITH, AND S. TROYANSKI \\ (Communicated by Thomas Schlumprecht)
}

\begin{abstract}
We show that if $X$ and $Y$ are Banach spaces, where $Y$ is separable and polyhedral, and if $T: X \rightarrow Y$ is a bounded linear operator such that $T^{*}\left(Y^{*}\right)$ contains a boundary $B$ of $X$, then $X$ is separable and isomorphic to a polyhedral space. Some corollaries of this result are presented.
\end{abstract}

\section{The MAIn ReSUlt}

Let $X$ be a Banach space. A subset $B \subset S_{X^{*}}$ of the unit sphere $S_{X^{*}}$ of $X$ is called a boundary of $X$ if, for any $x \in X$, there is $f \in B$ satisfying $f(x)=\|x\|$. From the Krein-Milman Theorem, it follows that the set ext $B_{X^{*}}$ of all extreme points of the unit ball $B_{X^{*}}$ of $X^{*}$ is a boundary. Easy examples show that a boundary may be a proper subset of ext $B_{X^{*}}$. A separation theorem shows that, for any boundary $B$, we have $w^{*}-\operatorname{cl}(\operatorname{co} B)=B_{X^{*}}$. It is clear that if $X$ is infinite-dimensional, then any boundary of $X$ must be infinite.

Recall that a Banach space $X$ is polyhedral if, given any finite-dimensional subspace $E \subseteq X$, the unit ball $B_{E}$ of $E$ is a polytope. If $X$ has a countably infinite boundary, then it is separable and isomorphic to a polyhedral space (see [F]).

The following theorem is the main result of this paper.

Theorem 1.1. Let $X$ and $Y$ be Banach spaces, where $Y$ is separable and polyhedral. Assume that $T: X \rightarrow Y$ is a bounded linear operator such that $T^{*}\left(Y^{*}\right)$ contains a boundary $B$ of $X$. Then $X$ is separable and isomorphic to a polyhedral space.

The proof of Theorem 1.1 uses the following result.

Proposition 1.2. Let $T: X \rightarrow Y$ be a linear bounded operator such that $T^{*}\left(Y^{*}\right)$ contains a boundary $B$ of $X$. Then $T^{*}\left(Y^{*}\right)$ is norm dense in $X^{*}$.

Proof. We make use of the so-called (I)-property (see $\left[\mathrm{FL}\right.$ ). Let $K \subset X^{*}$ be $w^{*}$-compact and convex, and suppose that $B \subset K$. We say that $B$ has the (I)-property if, whenever $B=\bigcup_{i=1}^{\infty} B_{i}$, where $B_{i} \subset B_{i+1}$, then the set $\bigcup_{i=1}^{\infty} w^{*}$ $\operatorname{cl}\left(\operatorname{co} B_{i}\right)$ is norm-dense in $K$. It is proved in FL that, for $K$ as above, any boundary $B$ of $K$ has the (I)-property ( $B \subset K$ is a boundary of $K$ if, for any $x \in X$, there is $f \in B$ such that $f(x)=\max x(K))$.

Received by the editors March 31, 2004 and, in revised form, September 8, 2014.

2010 Mathematics Subject Classification. Primary 46B20.

Key words and phrases. Polyhedral norms, renormings, boundaries, polytopes.

The first author was supported by Israel Science Foundation, Grant 209/09. The second and third authors were supported financially by Science Foundation Ireland under Grant Number 'SFI 11/RFP.1/MTH/3112'. The third author was also supported by FEDER-MCI MTM2011-22457 and by the Bulgarian National Scientific Fund DFNI-I02/10. 
Put $K=B_{X^{*}}$, and $B_{i}=T^{*}\left(i B_{Y^{*}}\right) \cap B$ for $i=1,2, \ldots$ Clearly, $\bigcup_{i=1}^{\infty} w^{*}$ $\operatorname{cl}\left(\operatorname{co} B_{i}\right) \subset T^{*}\left(Y^{*}\right)$, and the result follows.

Proof of Theorem 1.1. Without loss of generality, we assume that $\|T\|=1$. Since $Y$ is a separable polyhedral space, it follows that $Y^{*}$ is separable (see $[\mathrm{F}]$ ), and hence by Proposition [1.2, $X^{*}$ and $X$ are separable too. Next, it is easily seen that $T$ is injective. Also, without loss of generality, we can assume that $T$ is a dense embedding, i.e. $\operatorname{cl} T(X)=Y$ (if necessary, pass to the subspace $Y_{1}=\operatorname{cl} T(X)$ and the operator $T_{1}: X \rightarrow Y_{1}$ ). In particular, we can assume that $T^{*}$ is injective. Now set

$$
W_{n}=T^{*-1}\left(n T^{*}\left(B_{Y^{*}}\right) \cap B_{X^{*}}\right), \quad n=1,2, \ldots
$$

Clearly,

(recall that $\|T\|=1$ ).

$$
B_{Y^{*}} \subset W_{n} \subset n B_{Y^{*}}, \quad n=1,2, \ldots
$$

The $W_{n}$ are convex centrally symmetric $w^{*}$-compact bodies in $Y^{*}$ (with $Y$ separable and polyhedral). Choose a sequence $\left\{\varepsilon_{n}\right\}_{n=1}^{\infty}$ of positive numbers tending to 0 . By [DFH, Theorem 1.1], each such body $W_{n}$ can be approximated by a convex centrally symmetric $w^{*}$-compact body $A_{n}$ having a countable boundary, say $\left\{ \pm h_{i}^{n}\right\}_{i=1}^{\infty}$, such that

(a) $\left(1-\varepsilon_{n}\right) A_{n} \subset W_{n} \subset A_{n}$, and

(b) no $w^{*}$-limit point of $\left\{ \pm h_{i}^{n}\right\}_{i=1}^{\infty}$ is a support point of $A_{n}$, for any $y \in Y$.

Define

$$
\begin{gathered}
U^{*}=w^{*}-\operatorname{cl}\left(\operatorname{co}_{n=1}^{\infty}\left(1+\varepsilon_{n}\right) T^{*}\left(A_{n}\right)\right), t_{i}^{n}=T^{*} h_{i}^{n}, n=1,2, \ldots, \\
\tilde{B}=\left\{ \pm\left(1+\varepsilon_{n}\right) t_{i}^{n}\right\}_{i, n=1}^{\infty} .
\end{gathered}
$$

It is easily seen that $U^{*}$ is a convex centrally symmetric $w^{*}$-compact set in $X^{*}$, and $U^{*}=w^{*}-\operatorname{cl}(\operatorname{co} \tilde{B})$. Moreover, $B \subset U^{*}$ and hence $B_{X^{*}} \subset U^{*}$.

It follows that $U^{*}$, as the unit ball, defines an equivalent dual norm on $X^{*}$. We denote the corresponding norm on $X$ by $\|\cdot|\||$, and show that $(X,\||\cdot|\|)$ is polyhedral. To prove this statement, it is enough to check that $\tilde{B}$ has $(*)$ (see [FLP]), i.e. no $w^{*}$-limit point of $\tilde{B}$ is a support point of $U^{*}$, for any $x \in X$.

If $f$ is a $w^{*}$-limit point of $\tilde{B}$, then, by the separability of $X$, we may find a sequence of distinct points in $\tilde{B}$ that converges to $f$ in the $w^{*}$-topology. There are two possibilities. The first possibility is that, for some fixed $n$, we can write $f=w^{*}-\lim _{k}\left(1+\varepsilon_{n}\right) t_{i_{k}}^{n} \in\left(1+\varepsilon_{n}\right) T^{*}\left(A_{n}\right)$, where $i_{1}<i_{2}<\ldots$ Then $T^{*-1} f$ is a $w^{*}$-limit point of the set $\left\{ \pm\left(1+\varepsilon_{n}\right) h_{i}^{n}\right\}_{i=1}^{\infty}$ and, by (b) above, it cannot be a support point of $\left(1+\varepsilon_{n}\right) A_{n}$ for any $y \in Y$. Therefore, $f$ is not a support point of $\left(1+\varepsilon_{n}\right) T^{*}\left(A_{n}\right)$, for any $x \in X$. Since $f \in\left(1+\varepsilon_{n}\right) T^{*}\left(A_{n}\right) \subset U^{*}$ it follows that $f$ is not a support point of $U^{*}$, for any $x \in X$.

If the first possibility above does not hold, then we can write

$$
f=w^{*}-\lim _{k}\left(1+\varepsilon_{n_{k}}\right) t_{i_{k}}^{n_{k}},
$$

where $n_{k} \rightarrow \infty$ as $k \rightarrow \infty$. Assume to the contrary that $f$ is a support point of $U^{*}$ with $f\left(x_{0}\right)=\max x_{0}\left(U^{*}\right) \neq 0, x_{0} \in X$. By (a), it is easily seen that $f \in B_{X^{*}}$. Since $B_{X^{*}} \subset U^{*}$, it follows that $f\left(x_{0}\right)=\max x_{0}\left(B_{X^{*}}\right)=\left\|x_{0}\right\| \neq 0$. As $B$ is a boundary of $X$, there is $g \in B$ satisfying $g\left(x_{0}\right)=\left\|x_{0}\right\|$. Next, because $B \subset T^{*}\left(Y^{*}\right)$, it easily follows that $B \subset \bigcup_{n=1}^{\infty} T^{*}\left(A_{n}\right)$, and hence $g \in T^{*}\left(A_{m}\right)$ for 
some $m$. However, $\left(1+\varepsilon_{m}\right) g \in U^{*}$ implies $\max x_{0}\left(U^{*}\right) \geqslant\left(1+\varepsilon_{m}\right)\left\|x_{0}\right\|$, contradicting $\left\|x_{0}\right\|=f\left(x_{0}\right)=\max x_{0}\left(U^{*}\right) \neq 0$. The proof is complete.

\section{Consequences}

Corollary 2.1. Let $X$ be a Banach space and $Y_{i}, i=1,2, \ldots$, be a sequence of separable isomorphically polyhedral Banach spaces. Let $B$ be a boundary of $X$ and $T_{i}: X \rightarrow Y_{i}$ a sequence of bounded linear operators such that

$$
B \subset \bigcup_{i=1}^{\infty} T_{i}^{*}\left(Y_{i}^{*}\right)
$$

Then $X$ is separable and isomorphically polyhedral.

Proof. Without loss of generality we can assume that $Y_{i}$ is polyhedral and $\left\|T_{i}\right\|=1$ for all $i$. Let $Y=\left(\bigoplus_{i=1}^{\infty} Y_{i}\right)_{c_{0}}$. Clearly $Y$ is a separable isomorphically polyhedral space. Define $T: X \rightarrow Y$ by $(T x)_{i}=i^{-1} T_{i} x \in Y_{i}$. Evidently, $Y^{*}=\left(\bigoplus_{i=1}^{\infty} Y_{i}^{*}\right)_{\ell_{1}}$, and if $f \in Y^{*}$ and $x \in X$, we have

$$
\left(T^{*} f\right)(x)=f(T x)=\sum_{i=1}^{\infty} i^{-1} f_{i}\left(T_{i} x\right)=\sum_{i=1}^{\infty}\left(i^{-1} T_{i}^{*} f\right)(x),
$$

where $f=\left(f_{i}\right)_{i=1}^{\infty}$ and $\|f\|=\sum_{i=1}^{\infty}\left\|f_{i}\right\|$. In particular, if $g \in Y_{i}^{*} \subset Y^{*}$ we have $T^{*} g=i^{-1} T_{i}^{*} g$, whence

$$
B \subset \bigcup_{i=1}^{\infty} T_{i}^{*}\left(Y_{i}^{*}\right) \subset T^{*}\left(Y^{*}\right)
$$

Apply Theorem 1.1 to finish the proof.

Corollary 2.2. Assume that $X$ has a boundary $B$ which is contained in a set of the form

$$
\bigcup_{i=1}^{\infty} w^{*}-\operatorname{cl}\left(\operatorname{co} K_{i}\right)
$$

where the $K_{i}$ are countable $w^{*}$-compact subsets of $X^{*}$. Then $X$ is isomorphically polyhedral.

Proof. For $i \in \mathbb{N}$, let $Y_{i}$ be the isomorphically polyhedral space $C\left(K_{i}\right)$, and define $T_{i}: X \rightarrow Y_{i}$ by $\left(T_{i} x\right)(t)=t(x), x \in X, t \in K_{i}$. Then $w^{*}-\operatorname{cl}\left(\operatorname{co} K_{i}\right) \subset T^{*}\left(B_{C\left(K_{i}\right)^{*}}\right)$, and we can apply Corollary 2.1 .

We will see later in Remark 2.6 that we cannot drop the requirement that the $K_{i}$ above are $w^{*}$-compact. Our final result includes an application of the material above, stated in terms of M-bases.

Definition 2.3. Let $\left\{x_{i}\right\} \subset X$ be an M-basis of a Banach space $X$, having biorthogonal sequence $\left\{x_{i}^{*}\right\} \subset X^{*}$. We call a subset $A \subset X^{*}$ summable if $\sum_{i=1}^{\infty}\left|f\left(x_{i}\right)\right|<\infty$ for all $f \in A$.

Lemma 2.4. Let $B=\left\{ \pm f_{i}\right\} \subset S_{X^{*}}$ be a countable boundary of $X$, take a sequence of numbers $\left\{\varepsilon_{i}\right\}, 0<\varepsilon_{i}<\frac{1}{2}, \lim _{i} \varepsilon_{i}=0$, and a sequence of vectors $\left\{t_{i}\right\} \subset X^{*}$ satisfying $\left\|f_{i}-t_{i}\right\|<\varepsilon_{i}$. Then the sequence $\left\{ \pm h_{i}\right\}$, where $h_{i}=\left(1+2 \varepsilon_{i}\right) t_{i}, i=$ $1,2, \ldots$, is a boundary having $(*)$ with respect to the norm it generates, given by

$$
|\|x\||=\sup _{i}\left|h_{i}(x)\right|, \quad x \in X .
$$


Proof. First, note that for any $x \in X, x \neq 0$, we have

$$
\|x\| \mid\|x\| \text {. }
$$

Indeed, if $f_{i}(x)=\|x\|$, then

$$
\begin{aligned}
\|x\| & \geqslant\left(1+2 \varepsilon_{i}\right) t_{i}(x) \\
& \geqslant\left(1+2 \varepsilon_{i}\right) f_{i}(x)-\left(1+2 \varepsilon_{i}\right)\left\|t_{i}-f_{i}\right\|\|x\| \\
& \geqslant\left(1+2 \varepsilon_{i}\right)\left(1-\varepsilon_{i}\right)\|x\|>\|x\| .
\end{aligned}
$$

Put $V^{*}=\left\{f \in X^{*}:\|f\| \mid \leqslant 1\right\}, S_{V^{*}}=\partial V^{*}$. By using the Hahn-Banach Theorem and (1), we easily obtain $B_{X^{*}} \subset V^{*}$ and, again by using (1), we see that no functional $f \in S_{V^{*}} \cap B_{X^{*}}$ (if any) attains its norm with respect to $\|\cdot \cdot\|$. However, any $w^{*}$-limit point $g$ of $B_{1}$ satisfying $\|g\| \|=1$ (if any) lies in $B_{X^{*}}$ (recall that $\left.\lim _{i} \varepsilon_{i}=0\right)$. The proof is complete.

Theorem 2.5. For a separable Banach space $X$, the following assertions are equivalent:

(a) $X$ admits a boundary $B$ and a bounded linear operator $T: X \rightarrow c_{0}$ such that $T^{*}\left(c_{0}^{*}\right) \supset B$.

(b) $X$ admits a boundary $B$ and a bounded linear operator $T: X \rightarrow Y$ into a polyhedral space $Y$ such that $T^{*}\left(Y^{*}\right) \supset B$.

(c) $X$ is isomorphically polyhedral.

(d) $X$ admits an equivalent norm having a boundary $B$, which is summable with respect to a normalized $M$-basis $\left\{x_{i}\right\}$ with bounded biorthogonal sequence $\left\{x_{i}^{*}\right\}$.

Proof. (a) $\Rightarrow$ (b) is trivial, while (b) $\Rightarrow$ (c) is Theorem 1.1. To prove (c) $\Rightarrow$ (d), we can assume without loss of generality that $X$ polyhedral. By [F1], $X$ admits a countable boundary $\left\{f_{i}\right\}$, and $X^{*}$ is separable. It is well-known (see for instance [FHHMVZ, Theorem 4.59]) that $X$ admits a (shrinking) normalized M-basis $\left\{x_{i}\right\}$ with bounded biorthogonal sequence $\left\{x_{i}^{*}\right\}$. By using Lemma 2.4. we easily obtain a sequence $B=\left\{h_{i}\right\} \subset \operatorname{span}\left\{x_{i}^{*}\right\}$ which is a boundary of $X$ with respect to an equivalent norm. Clearly, $B$ is summable with respect to the M-basis $\left\{x_{i}\right\}$. Finally, we prove $(\mathrm{d}) \Rightarrow(\mathrm{a})$. Let $B$ be a boundary of $X$ which is summable with respect to a normalized M-basis $\left\{x_{i}\right\}$ with bounded biorthogonal sequence $\left\{x_{i}^{*}\right\}$. Define $T: X \rightarrow c_{0}$ by

$$
T x=\left(x_{i}^{*}(x)\right)_{i=1}^{\infty}, \quad x \in X .
$$

Evidently, $\|T\|=\sup _{i}\left\|x_{i}^{*}\right\|<\infty$. If $\left\{e_{i}\right\}$ is the natural basis of $\ell_{1}=c_{0}^{*}$, then it is easily seen that $T^{*} e_{i}=x_{i}^{*}, i=1,2, \ldots$ Since $B$ is summable, it follows that $T^{*}\left(c_{0}^{*}\right) \supset B$.

Remark 2.6. We cannot replace the $x_{i}^{*}$ in Theorem 2.5(d), even with a normalized basis of $X^{*}$, if its biorthogonal sequence does not belong to $X$. Indeed, if $X^{*}$ is isomorphic to $\ell_{1}$, then it admits a normalized basis, with respect to which every element of $X^{*}$ is an absolutely summable combination. However, there exist nonisomorphically polyhedral Banach spaces having duals isomorphic to $\ell_{1}$. The spaces in e.g. $\mathrm{BD}$ and $[\mathrm{AH}]$ have duals isomorphic to $\ell_{1}$ but do not contain any isomorphic copies of $c_{0}$, and in order for a Banach space to be isomorphically polyhedral, it 
is necessarily $c_{0}$-saturated $[\mathrm{F}$. In fact, any space $X$ having a separable dual space (many of which are not isomorphically polyhedral) embeds isomorphically into a space having a dual space isomorphic to $\ell_{1}$ [FOS. The same examples show that it is necessary for the $K_{i}$ in Corollary 2.2 to be $w^{*}$-compact.

\section{REFERENCES}

[AH] Spiros A. Argyros and Richard G. Haydon, A hereditarily indecomposable $\mathcal{L}_{\infty}$-space that solves the scalar-plus-compact problem, Acta Math. 206 (2011), no. 1, 1-54, DOI 10.1007/s11511-011-0058-y. MR2784662 (2012e:46031)

[BD] J. Bourgain and F. Delbaen, A class of special $\mathcal{L}_{\infty}$ spaces, Acta Math. 145 (1980), no. 3-4, 155-176, DOI 10.1007/BF02414188. MR590288 (82h:46023)

[DFH] Robert Deville, Vladimir Fonf, and Petr Hájek, Analytic and polyhedral approximation of convex bodies in separable polyhedral Banach spaces, Israel J. Math. 105 (1998), 139-154, DOI 10.1007/BF02780326. MR1639743 (99h:46006)

[FHHMVZ] Marián Fabian, Petr Habala, Petr Hájek, Vicente Montesinos, and Václav Zizler, Banach space theory, CMS Books in Mathematics/Ouvrages de Mathématiques de la SMC, Springer, New York, 2011. The basis for linear and nonlinear analysis. MR2766381 (2012h:46001)

[FOS] D. Freeman, E. Odell, and Th. Schlumprecht, The universality of $\ell_{1}$ as a dual space, Math. Ann. 351 (2011), no. 1, 149-186, DOI 10.1007/s00208-010-0601-8. MR2824850 (2012i:46019)

[F] V. P. Fonf, Some properties of polyhedral Banach spaces (Russian), Funktsional. Anal. i Prilozhen. 14 (1980), no. 4, 89-90. MR595744(82a:46017)

[F1] V. P. Fonf, Weakly extremal properties of Banach spaces (Russian), Mat. Zametki 45 (1989), no. 6, 83-92, 112, DOI 10.1007/BF01158239; English transl., Math. Notes 45 (1989), no. 5-6, 488-494. MR.1019040(90k:46032)

[FL] Vladimir P. Fonf and Joram Lindenstrauss, Boundaries and generation of convex sets, Israel J. Math. 136 (2003), 157-172, DOI 10.1007/BF02807196. MR.1998108 (2004g:46021)

[FLP] V. P. Fonf, J. Lindenstrauss, and R. R. Phelps, Infinite dimensional convexity, Handbook of the geometry of Banach spaces, Vol. I, North-Holland, Amsterdam, 2001, pp. 599-670, DOI 10.1016/S1874-5849(01)80017-6. MR1863703 (2003c:46014)

Department of Mathematics, Ben Gurion University of the Negev, Beer-Sheva, IsRAEL

E-mail address: fonf@math.bgu.ac.il

School of Mathematical and Statistical Sciences, University College Dublin, BelFIELD, DUblin 4, IRELAND

E-mail address: richard.smith@maths.ucd.ie

Departamento de Matemáticas, Universidad de Murcia, Campus de Espinardo, 30100 Espinardo (Murcia), Spain - and - Institute of Mathematics and Informatics, Bulgarian Academy of Science, Bl.8, ACAD. G. Bonchev Str. 1113 Sofia, Bulgaria

E-mail address: stroya@um.es 